\title{
A DENJOY-WOLFF THEOREM FOR COMPACT HOLOMORPHIC MAPPINGS IN COMPLEX BANACH SPACES
}

\author{
Monika Budzyńska, Tadeusz Kuczumow and Simeon Reich
}

Uniwersytet Marii Curie-Skłodowskiej, Instytut Matematyki 20-031 Lublin, Poland; monikab1@hektor.umcs.lublin.pl

Uniwersytet Marii Curie-Skłodowskiej, Instytut Matematyki 20-031 Lublin, Poland; tadek@hektor.umcs.lublin.pl

The Technion - Israel Institute of Technology, Department of Mathematics 32000 Haifa, Israel; sreich@tx.technion.ac.il

\begin{abstract}
We establish a Denjoy-Wolff theorem for compact holomorphic self-mappings of bounded and strictly convex domains in arbitrary complex Banach spaces.
\end{abstract}

\section{Introduction}

The following version of the Denjoy-Wolff theorem ([8], [33], [34] and [35]) for bounded and strictly convex domains in complex and reflexive Banach spaces has recently been established in [4] (see [3] and [5] for an up-to-date list of references regarding this topic).

Theorem 1.1. [4] If $D$ is a bounded and strictly convex domain in a complex and reflexive Banach space $(X,\|\cdot\|)$, and $f: D \mapsto D$ is compact, holomorphic and fixed-point-free, then there exists a point $\xi \in \partial D$ such that the sequence $\left\{f^{n}\right\}$ of the iterates of $f$ converges in the compact-open topology to the constant map taking the value $\xi$.

Since the assumption that the complex Banach space $(X,\|\cdot\|)$ is reflexive is essential in [4], it is natural to ask if Theorem 1.1 holds in all Banach spaces. In the present paper we answer this question in the affirmative. In other words, we extend Theorem 1.1 to the case of compact holomorphic (more generally, $k_{D}$-nonexpansive) self-mappings of bounded and strictly convex domains in arbitrary complex Banach spaces. We also show that the sequence $\left\{f^{n}\right\}$ of the iterates of $f$ converges to the constant map taking the value $\xi$ even in the bounded-open topology. Observe that in such a general setting, the main difficulty is that we cannot use either complex geodesics or reflexivity in the proofs of our results. We do, however, retain the compactness assumption on our self-mappings because in the setting of infinite-dimensional complex Banach spaces, the Denjoy-Wolff theorem fails even for biholomorphic self-mappings of the open unit ball in Hilbert space [32].

doi:10.5186/aasfm.2013.3846

2010 Mathematics Subject Classification: Primary 32A10, 46G20, 46T25, 47H09, 47H10.

Key words: Banach space, compact mapping, Denjoy-Wolff theorem, fixed point, holomorphic mapping, horosphere, iterates of holomorphic mappings, $k_{D}$-nonexpansive mapping, Kobayashi distance, strictly convex domain. 
Our paper is organized as follows. The next section contains several preliminaries concerning the Kobayashi distance and holomorphic mappings in Banach spaces. See, for instance, Theorems 2.3 and 2.4. Section 3 is devoted to horospheres (see Theorems 3.1-3.3). Finally, two versions of our Denjoy-Wolff theorem are stated and proved in Section 4 (see Theorems 4.1 and 4.2). This section also contains three consequences of these theorems (Corollaries 4.3-4.5).

\section{The Kobayashi distance $k_{D}$, holomorphic mappings and $k_{D}$-nonexpansive mappings}

Let $(X,\|\cdot\|)$ be an arbitrary complex Banach space. Throughout this paper all domains $D \subset X$ are bounded and convex.

In this part of our paper we recall a few properties of the Kobayashi distance $k_{D}$ ([23], [24], [25]), which are common to all bounded and convex domains in complex Banach spaces (for more properties of $k_{D}$ see [27]).

We begin with the definition of the Poincaré distance. Let $\Delta$ be the open unit disc in the complex plane $\mathbf{C}$. Recall that the Poincaré distance $\rho_{\Delta}$ on $\Delta$ is given by

$$
k_{\Delta}(z, w)=\rho_{\Delta}(z, w):=\arg \tanh \left|\frac{z-w}{1-z \bar{w}}\right|=\arg \tanh (1-\sigma(z, w))^{\frac{1}{2}},
$$

where

$$
\sigma(z, w)=\frac{\left(1-|z|^{2}\right)\left(1-|w|^{2}\right)}{|1-z \bar{w}|^{2}}, \quad z, w \in \Delta .
$$

Next, for any $a \in \Delta$, consider the Möbius transformation $m_{a}: \Delta \mapsto \Delta$ defined by

$$
m_{a}(z):=\frac{z+a}{1+z \bar{a}},: z \in \Delta .
$$

This mapping is not only a biholomorphic automorphism of $\Delta$, but also a $k_{\Delta}$-isometry.

We also know that for each pair of distinct points $x, y \in \Delta$, there exist a unique geodesic line in $\left(\Delta, k_{\Delta}\right)$ passing through them and a unique geodesic segment $[x, y]$ joining them, i.e., for each $0 \leq t \leq 1$, there is a unique point $z=(1-t) x \oplus$ ty satisfying $k_{\Delta}(x, z)=t k_{\Delta}(x, y)$ and $k_{\Delta}(z, y)=(1-t) k_{\Delta}(x, y)$. For each $0 \neq z \in \Delta$, the segment $\left\{x \in \Delta\right.$ : there exists $t \in(-1,1) \subset \mathbf{R}$ such that $\left.x=t \frac{z}{\|z\|}\right\}$ is the unique geodesic line passing through 0 and $z$ ([16], [9]).

Now let $D$ be a bounded and convex domain in a complex Banach space $(X,\|\cdot\|)$. We use the following definition of the Kobayashi distance on $D$. This definition is, in fact, the definition of the Lempert function $\delta$ ([30], [11]):

$$
\begin{gathered}
k_{D}(x, y)=\delta_{D}(x, y):=\inf \left\{\rho_{\Delta}(0, \lambda): \lambda \in[0,1) \text { and there exists } F \in H(\Delta, D)\right. \\
\text { so that } F(0)=x, F(\lambda)=y\} .
\end{gathered}
$$

It is known that the Kobayashi distance $k_{D}$ is locally equivalent to the norm $\|\cdot\|$ in $X[18]$.

We also recall the following result.

Lemma 2.1. [21], [28], [29] Let $D$ be a bounded and convex domain in a complex Banach space $(X,\|\cdot\|)$.

(i) If $x, y, w, z \in D$ and $s \in[0,1]$, then

$$
k_{D}(s x+(1-s) y, s w+(1-s) z) \leq \max \left[k_{D}(x, w), k_{D}(y, z)\right]
$$


(ii) if $x, y \in D$ and $s, t \in[0,1]$, then

$$
k_{D}(s x+(1-s) y, t x+(1-t) y) \leq k_{D}(x, y) .
$$

This result shows, for example, that our horospheres are convex (see Theorem 3.1(i) below). It is also used in the proof of Theorem 3.2.

In order to recall a characterization of $k_{D^{-}}$-bounded sets, we need the following notion.

Definition 2.1. [18] Let $D$ be a bounded and convex domain in a complex Banach space $(X,\|\cdot\|)$. A nonempty subset $C$ of $D$ is said to lie strictly inside $D$ if

$$
\operatorname{dist}_{\|\cdot\|}(C, \partial D):=\inf \{\|x-y\|: x \in C, y \in \partial D\}>0 .
$$

Let $D$ be a bounded and convex domain in a complex Banach space $(X,\|\cdot\|)$. It is known that a nonempty subset $C$ of $D$ is $k_{D}$-bounded if and only if $C$ lies strictly inside $D[18]$.

Next we recall the definition of strict convexity.

Definition 2.2. [15] We say that a bounded and convex domain $D$ in a complex Banach space $(X,\|\cdot\|)$ is strictly convex if for each $x, y \in \bar{D}^{\|\cdot\|}$, the open segment

$$
(x, y)=\{z \in X: z=s x+(1-s) y \text { for some } 0<s<1\}
$$

lies in $D$.

Using strict convexity, one can prove the following very useful lemma.

Lemma 2.2. [21], [27] Let $D$ be a bounded and strictly convex domain in a complex Banach space $(X,\|\cdot\|)$. Let $\left\{x_{j}\right\}_{j \in J}$ and $\left\{y_{j}\right\}_{j \in J}$ be two nets in $D$ which converge in norm to $\xi \in \partial D$ and to $\eta \in \bar{D}^{\|\cdot\|}$, respectively. If

$$
\sup \left\{k_{D}\left(x_{j}, y_{j}\right): j \in J\right\}=c<\infty,
$$

then $\xi=\eta$.

The notion of a nonexpansive (that is, 1-Lipschitz) mapping plays a key role in our paper. This is due to the fact that if $D_{1}$ and $D_{2}$ are bounded domains in the complex Banach spaces $\left(X_{1},\|\cdot\|_{1}\right)$ and $\left(X_{2},\|\cdot\|_{2}\right)$, respectively, and $k_{D_{1}}$ and $k_{D_{2}}$ are the Kobayashi distances on $D_{1}$ and $D_{2}$, respectively, then each holomorphic mapping $f: D_{1} \mapsto D_{2}$ is nonexpansive, i.e.,

$$
k_{D_{2}}(f(x), f(y)) \leq k_{D_{1}}(x, y)
$$

for all $x, y \in D_{1}$ [18] (see also [9], [14], [16], [19] and [31]).

In particular, if $D$ is a bounded domain in a complex Banach space $(X,\|\cdot\|)$, then each holomorphic self-mapping $f: D \mapsto D$ is $k_{D}$-nonexpansive.

Let $D$ be a bounded domain in a complex Banach space $(X,\|\cdot\|)$. A mapping $f: D \mapsto D$ is said to map $D$ strictly inside $D$ if $f(D)$ lies strictly inside $D$. Such a mapping is a strict $k_{D}$-contraction according to the Earle-Hamilton theorem [12] (see also [14]).

Theorem 2.3. [12] Let $D$ be a bounded domain in a complex Banach space $(X,\|\cdot\|)$. If a holomorphic $f: D \mapsto D$ maps $D$ strictly inside itself, then there exists a number $0 \leq t<1$ such that

$$
k_{D}(f(x), f(y)) \leq t k_{D}(x, y)
$$


for all $x$ and $y$ in $D$.

Hence, if $D$ is a bounded and convex domain in a Banach space $(X,\|\cdot\|)$, then by the Earle-Hamilton theorem, the mapping $g_{s, z}(\cdot):=(1-s) z+s(\cdot): D \mapsto D$ is a strict $k_{D}$-contraction for each $z \in D$ and $0 \leq s<1$. Thus for each $k_{D}$-nonexpansive mapping $f: D \mapsto D$, the mapping $f_{s, z}:=g_{s, z} \circ f=(1-s) z+s f: D \mapsto D$ is a strict $k_{D^{-}}$-contraction and has exactly one fixed point, which we denote by $h_{f}(s, z)$. Fix $0 \leq s<1$ and $x_{0} \in D$. Then the mapping $h_{f}(s, \cdot): D \mapsto D$ is $k_{D}$-nonexpansive (holomorphic if $f$ is holomorphic [7], [14] and [17]) as the limit of the sequence $\left\{f_{s, \cdot}^{n}\left(x_{0}\right)\right\}$.

Finally, we recall the definition of a compact mapping.

Definition 2.3. Let $D$ be a bounded and convex domain in a complex Banach space $(X,\|\cdot\|)$. If $f: D \mapsto D$ and the norm closure of the set $f(D)$ is compact in $(X,\|\cdot\|)$, then we say that $f$ is a compact mapping.

Using Całka's theorem [6], one arrives at the following basic result.

Theorem 2.4. [28] (see also [20], [21] and [26]) Let $D$ be a bounded and convex domain in a complex Banach space $(X,\|\cdot\|)$. If $f: D \mapsto D$ is $k_{D}$-nonexpansive and compact, then the following statements are equivalent:

(i) $f$ has a fixed point;

(ii) there exist a point $x \in D$ and a $k_{D}$-bounded subsequence $\left\{f^{n_{i}}(x)\right\}$ of its iterates;

(iii) there exists a point $x \in D$ such that $\left\{f^{n}(x)\right\}$ is $k_{D^{-b o u n d e d}}$;

(iv) for each point $x \in D$, the sequence $\left\{f^{n}(x)\right\}$ of its iterates is $k_{D^{-}}$-bounded;

(v) there exists a nonempty, $k_{D}$-closed, convex, $k_{D}$-bounded and $f$-invariant subset $C$ of $D$;

(vi) there exists a nonempty, $k_{D}$-bounded and $f$-invariant subset $C$ of $D$;

(vii) there exists a $k_{D}$-bounded and norm convergent sequence $\left\{x_{n}\right\}$ with $\left(f\left(x_{n}\right)-x_{n}\right) \rightarrow 0$

(viii) there exists a $k_{D}$-bounded sequence $\left\{x_{n}\right\}$ such that $\left(f\left(x_{n}\right)-x_{n}\right) \rightarrow 0$.

\section{Horospheres}

The main tool in the proof of our Denjoy-Wolff theorem (see Section 4) is the horosphere $H\left(x, \xi, R,\left\{x_{n}\right\}\right)$. To define it, we first recall that the set $\mathbf{N}$ of natural numbers can be treated as a sequence $\{n\}_{n \in \mathbf{N}}$. Hence it has a subnet $\left\{n_{\gamma}\right\}_{\gamma \in \Gamma}$ which is an ultranet (see [13] and [22] for properties of ultranets). We fix, once and for all, such a subnet $\left\{n_{\gamma}\right\}_{\gamma \in \Gamma}$. Now we define the horosphere $H\left(x, \xi, R,\left\{x_{n}\right\}\right)$ in the following way.

Definition 3.1. [5] (see also [21], [27] and [29]) Let $D$ be a bounded and convex domain in an arbitrary complex Banach space $(X,\|\cdot\|)$. Let $x \in D, \xi \in \partial D$, $R>0, x_{n} \in D, n=1,2, \ldots$, and $\lim _{n \rightarrow \infty} x_{n}=\xi$. The horosphere $H\left(x, \xi, R,\left\{x_{n}\right\}\right)$ in $D$ is defined as follows:

$$
H\left(x, \xi, R,\left\{x_{n}\right\}\right):=\left\{y \in D: \lim _{\gamma \in \Gamma}\left[k_{D}\left(y, x_{n_{\gamma}}\right)-k_{D}\left(x, x_{n_{\gamma}}\right)\right]<\frac{1}{2} \log R\right\} .
$$

The following properties of the horospheres $H\left(x, \xi, R,\left\{x_{n}\right\}\right)$ are known. 
Theorem 3.1. [4] Let $D$ be a bounded and convex domain in a complex Banach space $(X,\|\cdot\|)$. Let $x \in D, \xi \in \partial D, R>0, x_{n} \in D, n=1,2, \ldots$, and $\lim _{n \rightarrow \infty} x_{n}=\xi$. Then the horospheres $H\left(x, \xi, R,\left\{x_{n}\right\}\right)$ have the following properties:

(i) if the horosphere $H\left(x, \xi, R,\left\{x_{n}\right\}\right)$ is nonempty, then it is convex;

(ii) for every $0<R_{1}<R_{2}$, we have

$$
\left[\overline{\left.H\left(x, \xi, R_{1},\left\{x_{n}\right\}\right)^{\|\cdot\|} \cap D\right] \subset H\left(x, \xi, R_{2},\left\{x_{n}\right\}\right) ;}\right.
$$

(iii) if $x, \tilde{x} \in D$ and $\lim _{\gamma \in \Gamma}\left[k_{D}\left(\tilde{x}, x_{n_{\gamma}}\right)-k_{D}\left(x, x_{n_{\gamma}}\right)\right]<\frac{1}{2} \log L$, then

$$
H\left(\tilde{x}, \xi, R,\left\{x_{n}\right\}\right) \subset H\left(x, \xi, L R,\left\{x_{n}\right\}\right) ;
$$

(iv) for every $R>1$, we have $B\left(x, \frac{1}{2} \log R\right) \subset H\left(x, \xi, R,\left\{x_{n}\right\}\right)$, where $B\left(x, \frac{1}{2} \log R\right)$ is a ball in the metric space $\left(D, k_{D}\right)$;

(v) for every $R<1$, we have $B\left(x,-\frac{1}{2} \log R\right) \cap H\left(x, \xi, R,\left\{x_{n}\right\}\right)=\emptyset$, where $B\left(x,-\frac{1}{2} \log R\right)$ is, once again, a ball in the metric space $\left(D, k_{D}\right)$;

(vi) $\bigcup_{R>0} H\left(x, \xi, R,\left\{x_{n}\right\}\right)=D$ and $\bigcap_{R>0} H\left(x, \xi, R,\left\{x_{n}\right\}\right)=\emptyset$;

(vii) $\bigcap_{R>0} \overline{H\left(x, \xi, R,\left\{x_{n}\right\}\right)^{\|\cdot\|}} \subset \partial D$;

(viii) if $D$ is strictly convex and if the intersection $\left.\bigcap_{R>0} \overline{H\left(x, \xi, R,\left\{x_{n}\right\}\right.}\right)^{\|\cdot\|}$ is nonempty, then $\bigcap_{R>0} \overline{H\left(x, \xi, R,\left\{x_{n}\right\}\right)^{\|\cdot\|}}=\{\xi\}$.

Observe that directly from (iii) we get that if for some $\tilde{x} \in D$ all the horospheres $H\left(\tilde{x}, \xi, R,\left\{x_{n}\right\}\right)$ are nonempty, then the same is true when $\tilde{x}$ is replaced with any $x \in D$. We do not know, however, whether for each $R>0$, the horosphere $H\left(x, \xi, R,\left\{x_{n}\right\}\right)$ is always nonempty. Recall that this is valid for open unit balls in complex Banach spaces ([5], [20], [21], [26], [27], [29]; see also [1], [2] and [3] for the case of bounded and convex domains in $\mathbf{C}^{k}$ ). Now we prove this fact in the special case where the sequence $\left\{x_{n}\right\}$ stems from a compact, fixed-point-free and $k_{D}$-nonexpansive self-mapping $f$ of $D$; in particular, from a compact, fixed-point-free and holomorphic self-mapping of $D$. In contrast with [4], we no longer assume that the complex Banach space $X$ is reflexive.

Theorem 3.2. Let $D$ be a bounded and convex domain in an arbitrary complex Banach space $(X,\|\cdot\|)$. Let $f: D \mapsto D$ be a compact, $k_{D}$-nonexpansive and fixedpoint-free mapping. Fix $\tilde{x} \in D$ and an arbitrary sequence $\left\{z_{n}\right\} \subset D$. Let the sequences $\left\{s_{n}\right\} \subset \mathbf{R}$ and $\left\{x_{n}\right\} \subset D$ be such that

$$
0<s_{n}<1
$$

for each $n \in \mathbf{N}$,

$$
\begin{aligned}
\lim _{n \rightarrow \infty} s_{n} & =1, \\
x_{n} & =x_{n}\left(s_{n}, z_{n}\right)=h_{f}\left(s_{n}, z_{n}\right)=f_{s_{n}, z_{n}}\left(x_{n}\left(s_{n}, z_{n}\right)\right) \\
& =\left(1-s_{n}\right) z_{n}+s_{n} f\left(x_{n}\left(s_{n}, z_{n}\right)\right)=\left(1-s_{n}\right) z_{n}+s_{n} f\left(x_{n}\right)
\end{aligned}
$$

for each $n \in \mathbf{N}$, and

$$
\lim _{n \rightarrow \infty} x_{n}=\xi \in \partial D .
$$

Then the horosphere $H\left(\tilde{x}, \xi, R,\left\{x_{n}\right\}\right)$ is nonempty for each $R>0$. 
Proof. Directly from the definition of the Lempert function, we get that for each $w \in D, w \neq \tilde{x}$, and for each $\varepsilon>0$, there exist a holomorphic function $F_{w, \tilde{x}, \varepsilon}: \Delta \mapsto D$ and a real number $0<t_{w, \tilde{x}, \varepsilon}<1$ such that $F_{w, \tilde{x}, \varepsilon}(0)=\tilde{x}, F_{w, \tilde{x}, \varepsilon}\left(t_{w, \tilde{x}, \varepsilon}\right)=w$, and

$$
k_{D}(\tilde{x}, w)=k_{D}\left(F_{w, \tilde{x}, \varepsilon}(0), F_{w, \tilde{x}, \varepsilon}\left(t_{w, \tilde{x}, \varepsilon}\right)\right) \leq k_{\Delta}\left(0, t_{w, \tilde{x}, \varepsilon}\right)<k_{D}(\tilde{x}, w)+\varepsilon .
$$

Let $0<\tilde{\alpha}<k_{D}(\tilde{x}, w)$ and take $0<\tilde{t}_{w, \tilde{x}, \tilde{\alpha}}<t_{w, \tilde{x}, \varepsilon}$ such that $k_{\Delta}\left(0, \tilde{t}_{w, \tilde{x}, \tilde{\alpha}}\right)=\tilde{\alpha}$. Then we have

$$
\tilde{\alpha}+k_{\Delta}\left(\tilde{t}_{w, \tilde{x}, \tilde{\alpha}}, t_{w, \tilde{x}, \varepsilon}\right)=k_{\Delta}\left(0, \tilde{t}_{w, \tilde{x}, \tilde{\alpha}}\right)+k_{\Delta}\left(\tilde{t}_{w, \tilde{x}, \tilde{\alpha}}, t_{w, \tilde{x}, \varepsilon}\right)=k_{\Delta}\left(0, t_{w, \tilde{x}, \varepsilon}\right)<k_{D}(\tilde{x}, w)+\varepsilon
$$

and therefore

$$
k_{\Delta}\left(\tilde{t}_{w, \tilde{x}, \tilde{\alpha}}, t_{w, \tilde{x}, \varepsilon}\right)<k_{D}(\tilde{x}, w)+\varepsilon-\tilde{\alpha} .
$$

Now set $y_{w, \tilde{x}, \tilde{\alpha}, \varepsilon}:=F_{w, \tilde{x}, \varepsilon}\left(\tilde{t}_{w, \tilde{x}, \tilde{\alpha}}\right)$. Observe that

$$
\begin{aligned}
k_{D}\left(y_{w, \tilde{x}, \tilde{\alpha}, \varepsilon}, w\right) & =k_{D}\left(F_{w, \tilde{x}, \varepsilon}\left(\tilde{t}_{w, \tilde{x}, \tilde{\alpha}}\right), F_{w, \tilde{x}, \varepsilon}\left(t_{w, \tilde{x}, \varepsilon}\right)\right) \\
& \leq k_{\Delta}\left(\tilde{t}_{w, \tilde{x}, \tilde{\alpha}}, t_{w, \tilde{x}, \varepsilon}\right)<k_{D}(\tilde{x}, w)+\varepsilon-\tilde{\alpha}
\end{aligned}
$$

and

$$
k_{D}\left(\tilde{x}, y_{w, \tilde{x}, \tilde{\alpha}, \varepsilon}\right)=k_{D}\left(F_{w, \tilde{x}, \varepsilon}(0), F_{w, \tilde{x}, \varepsilon}\left(\tilde{t}_{w, \tilde{x}, \tilde{\alpha}}\right)\right) \leq k_{\Delta}\left(0, \tilde{t}_{w, \tilde{x}, \tilde{\alpha}}\right)=\tilde{\alpha} .
$$

Next fix $R>0$ and let $\alpha>\max \left\{-\frac{1}{2} \log R, 0\right\}$. Since $\lim _{n \rightarrow \infty} x_{n}=\xi \in \partial D$, we can assume, without any loss of generality, that $0<\alpha<k_{D}\left(\tilde{x}, x_{n}\right)$ for each $n \in \mathbf{N}$. Then for each point $y_{x_{n}, \tilde{x}, \alpha, \frac{1}{n}}$, we have

$$
k_{D}\left(y_{x_{n}, \tilde{x}, \alpha, \frac{1}{n}}, x_{n}\right)<k_{D}\left(\tilde{x}, x_{n}\right)-\alpha+\frac{1}{n}
$$

and therefore

$$
k_{D}\left(y_{x_{n}, \tilde{x}, \alpha, \frac{1}{n}}, x_{n}\right)-k_{D}\left(\tilde{x}, x_{n}\right)<-\alpha+\frac{1}{n} .
$$

For each $n \in \mathbf{N}$, set

$$
z_{x_{n}, \tilde{x}, \alpha}:=\left(1-s_{n}\right) z_{n}+s_{n} f\left(y_{x_{n}, \tilde{x}, \alpha, \frac{1}{n}}\right)
$$

Since (see Lemma 2.1)

$$
\begin{aligned}
& k_{D}\left(\left(1-s_{n}\right) z_{n}+s_{n} f(\tilde{x}), z_{x_{n}, \tilde{x}, \alpha}\right) \\
& =k_{D}\left(\left(1-s_{n}\right) z_{n}+s_{n} f(\tilde{x}),\left(1-s_{n}\right) z_{n}+s_{n} f\left(y_{x_{n}, \tilde{x}, \alpha, \frac{1}{n}}\right)\right) \\
& \leq k_{D}\left(\tilde{x}, y_{x_{n}, \tilde{x}, \alpha, \frac{1}{n}}\right) \leq \alpha
\end{aligned}
$$

and

$$
\lim _{n}\left[\left(1-s_{n}\right) z_{n}+s_{n} f(\tilde{x})\right]=f(\tilde{x}),
$$

the sequence $\left\{z_{x_{n}, \tilde{x}, \alpha}\right\}_{n \in \mathbf{N}}$ lies strictly inside $D$. Next, we note that

$$
\lim _{n}\left\|z_{x_{n}, \tilde{x}, \alpha}-f\left(y_{x_{n}, \tilde{x}, \alpha, \frac{1}{n}}\right)\right\|=0,
$$

where the sequence $\left\{f\left(y_{x_{n}, \tilde{x}, \alpha, \frac{1}{n}}\right)\right\}_{n \in \mathbf{N}}$ lies in the compact set $\overline{f(D)}\|\cdot\|$. This implies that the ultranet $\left\{z_{x_{n_{\gamma}}, \tilde{x}, \alpha}\right\}_{\gamma \in \Gamma}$ is convergent in the norm topology to a limit point $z_{\tilde{x}, \alpha} \in D$. Hence we have

$$
\lim _{\gamma \in \Gamma} k_{D}\left(z_{x_{n_{\gamma}}, \tilde{x}, \alpha}, z_{\tilde{x}, \alpha}\right)=0
$$


and

$$
\begin{aligned}
& \lim _{\gamma \in \Gamma}\left[k_{D}\left(z_{\tilde{x}, \alpha}, x_{n_{\gamma}}\right)-k_{D}\left(\tilde{x}, x_{n_{\gamma}}\right)\right]=\lim _{\gamma \in \Gamma}\left[k_{D}\left(z_{x_{n_{\gamma}}, \tilde{x}, \alpha}, x_{n_{\gamma}}\right)-k_{D}\left(\tilde{x}, x_{n_{\gamma}}\right)\right] \\
& =\lim _{\gamma \in \Gamma}\left[k_{D}\left(\left(1-s_{n_{\gamma}}\right) z_{n_{\gamma}}+s_{n_{\gamma}} f\left(y_{x_{n_{\gamma}}, \tilde{x}, \alpha, \frac{1}{n_{\gamma}}}\right),\left(1-s_{n_{\gamma}}\right) z_{n_{\gamma}}+s_{n_{\gamma}} f\left(x_{n_{\gamma}}\right)\right)-k_{D}\left(\tilde{x}, x_{n_{\gamma}}\right)\right] \\
& \leq \lim _{\gamma \in \Gamma} k_{D}\left[\left(y_{x_{n_{\gamma}}, \tilde{x}, \alpha, \frac{1}{n_{\gamma}}}, x_{n_{\gamma}}\right)-k_{D}\left(\tilde{x}, x_{n_{\gamma}}\right)\right] \leq \lim _{\gamma \in \Gamma}\left(-\alpha+\frac{1}{n_{\gamma}}\right)=-\alpha<\frac{1}{2} \log R
\end{aligned}
$$

(see Lemma 2.1). This means that the point $z_{\tilde{x}, \alpha}$ belongs to $H\left(\tilde{x}, \xi, R,\left\{x_{n}\right\}\right)$ and so the horosphere $H\left(\tilde{x}, \xi, R,\left\{x_{n}\right\}\right)$ is indeed not empty, as claimed.

Using now Theorem 3.1 and the beginning of the proof of Theorem 4.1 in [4], we get the following result which is crucial for the proof of our main theorem (Theorem 4.1 below).

Theorem 3.3. Let $D$ be a bounded and strictly convex domain in an arbitrary complex Banach space $(X,\|\cdot\|)$ and let $f: D \mapsto D$ be compact, $k_{D}$-nonexpansive and fixed-point-free. Let $\left\{z_{n}\right\}$ be an arbitrary sequence in $D$ and let the sequences $\left\{s_{n}\right\} \subset \mathbf{R}$ and $\left\{x_{n}\right\} \subset D$ be such that

$$
0<s_{n}<1
$$

for each $n \in \mathbf{N}$,

$$
\begin{aligned}
\lim _{n \rightarrow \infty} s_{n} & =1, \\
x_{n} & =x_{n}\left(s_{n}, z_{n}\right)=h_{f}\left(s_{n}, z_{n}\right)=f_{s_{n}, z_{n}}\left(x_{n}\left(s_{n}, z_{n}\right)\right) \\
& =\left(1-s_{n}\right) z_{n}+s_{n} f\left(x_{n}\left(s_{n}, z_{n}\right)\right)=\left(1-s_{n}\right) z_{n}+s_{n} f\left(x_{n}\right)
\end{aligned}
$$

for each $n \in \mathbf{N}$, and

$$
\lim _{n \rightarrow \infty} x_{n}=\xi \in \partial D .
$$

Then each horosphere $H\left(x, \xi, R,\left\{x_{n}\right\}\right)$ is nonempty and $f$-invariant, and the intersection

$$
\bigcap_{R>0} \overline{H\left(x, \xi, R,\left\{x_{n}\right\}\right)}\|\cdot\|=\bigcap_{R>0} \overline{H\left(x, \xi, R,\left\{x_{n}\right\}\right)}\|\cdot\|
$$

is the singleton $\{\xi\}$.

\section{A Denjoy-Wolff theorem}

We are now ready to formulate and establish the main theorem of our paper.

Theorem 4.1. If $D$ is a bounded and strictly convex domain in an arbitrary complex Banach space $(X,\|\cdot\|)$, and $f: D \mapsto D$ is compact, $k_{D \text {-nonexpansive and }}$ fixed-point-free, then there exists a point $\xi \in \partial D$ such that the sequence $\left\{f^{n}\right\}$ of the iterates of $f$ converges in the bounded-open topology to the constant map taking the value $\xi$, that is, the sequence $\left\{f^{n}\right\}$ tends to $\xi$, uniformly on each $k_{D}$-bounded subset $C$ of $D$.

Proof. Since $f$ is fixed-point-free and $k_{D}$-nonexpasive, and $\overline{f(D)}\|\cdot\|$ is a normcompact set, the Earle-Hamilton theorem (Theorem 2.3) and Theorem 2.4 yield a sequence

$$
\left\{x_{n}\right\}=\left\{x_{n}\left(s_{n}, z_{n}\right)\right\}=\left\{h_{f}\left(s_{n}, z_{n}\right)\right\}=\left\{f_{s_{n}, z_{n}}\left(x_{n}\right)\right\}=\left\{\left(1-s_{n}\right) z_{n}+s_{n} f\left(x_{n}\right)\right\}
$$


(where $z_{n} \in D, 0<s_{n}<1$ for $n=1,2, \ldots$, and $\lim _{n \rightarrow \infty} s_{n}=1$ ), which converges to a point $\xi \in \partial D$. By Theorem 3.3, for each $R>0$, the closure in $\left(D, k_{D}\right)$ of the horosphere $H\left(x, \xi, R,\left\{x_{n}\right\}\right)$ is a nonempty and $f$-invariant set. Next, since $f$ is fixed-point-free, Theorem 2.4 implies that for each $z \in D$, we have

$$
\lim _{n \rightarrow \infty} k_{D}\left(f^{n}(z), z\right)=\infty .
$$

Let $\emptyset \neq A \subset \partial D$ denote the set of all accumulation points of the sequence $\left\{f^{n}(z)\right\}$. By Lemma 2.2, the set $A$ is independent of the choice of $z \in D$ and therefore by Theorem 3.3 we get

$$
\emptyset \neq A \subset \partial D \cap \bigcap_{R>0} \overline{H\left(x, \xi, R,\left\{x_{n}\right\}\right)}\|\cdot\|
$$

where $\xi \in \partial D$. The equality $A=\{\xi\}$ implies that $\lim _{n \rightarrow \infty} f^{n}(z)=\xi$.

Now take an arbitrary norm-convergent sequence $\left\{f^{n_{i}}\left(x_{i}\right)\right\}$, where $n_{i} \rightarrow \infty$ as $i \rightarrow \infty$, and $\left\{x_{i}\right\}$ is a $k_{D}$-bounded sequence in $D$. Then the sequence $\left\{f\left(x_{i}\right)\right\}$ is

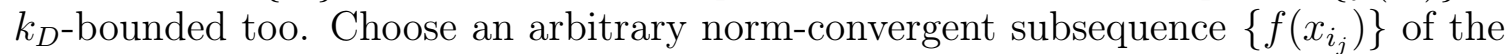
sequence $\left\{f\left(x_{i}\right)\right\}$ (recall that the mapping $f$ is compact) and $\operatorname{let} \lim _{j \rightarrow \infty} f\left(x_{i_{j}}\right)=z \in$ $D$. Observe that

$$
k_{D}\left(f^{n_{i_{j}}}\left(x_{i_{j}}\right), f^{n_{i_{j}}-1}(z)\right)=k_{D}\left(f^{n_{i_{j}}-1}\left(f\left(x_{i_{j}}\right)\right), f^{n_{i_{j}}-1}(z)\right) \leq k_{D}\left(f\left(x_{i_{j}}\right), z\right)
$$

for all $j \in \mathbf{N}$. Hence

$$
\sup _{j} k_{D}\left(f^{n_{i_{j}}}\left(x_{i_{j}}\right), f^{n_{i_{j}}-1}(z)\right)<\infty .
$$

Since $\lim _{i \rightarrow \infty} f^{n_{i}-1}(z)=\xi$, Lemma 2.2 implies that $\lim _{j \rightarrow \infty} f^{n_{i_{j}}}\left(x_{i_{j}}\right)=\xi$. This means that the sequence $\left\{f^{n_{i}}\left(x_{i}\right)\right\}$ also tends to $\xi$ as $i \rightarrow \infty$. Therefore the sequence $\left\{f^{n}\right\}$ of the iterates of $f$ converges in the bounded-open topology to the constant map taking the value $\xi$. This completes the proof of Theorem 4.1.

Since any holomorphic self-mapping $f$ of $D$ is $k_{D}$-nonexpansive, Theorem 4.1 immediately yields the following version of our Denjoy-Wolff theorem for bounded and strictly convex domains in an arbitrary complex Banach space.

Theorem 4.2. If $D$ is a bounded and strictly convex domain in an arbitrary complex Banach space $(X,\|\cdot\|)$, and $f: D \mapsto D$ is compact, holomorphic and fixedpoint-free, then there exists a point $\xi \in \partial D$ such that the sequence $\left\{f^{n}\right\}$ of the iterates of $f$ converges in the bounded-open topology to the constant map taking the value $\xi$.

We call the point $\xi \in \partial D$, obtained in Theorems 4.1 and 4.2 , the Wolff point of $f$.

Now, as in [4], we obtain the following three corollaries.

Corollary 4.3. Let $D$ be a bounded and strictly convex domain in an arbitrary complex Banach space $(X,\|\cdot\|)$, and let $f: D \mapsto D$ be compact, holomorphic $\left(k_{D^{-}}\right.$ nonexpansive) and fixed-point-free. Let $\left\{z_{j}\right\}$ be an arbitrary sequence in $D$ and let the sequence $\left\{x_{j}\right\} \subset D$ be such that

$$
\left\{x_{j}\right\}=\left\{h_{f}\left(s_{j}, z_{j}\right)\right\}=\left\{f_{s_{j}, z_{j}}\left(x_{j}\right)\right\}=\left\{\left(1-s_{j}\right) z_{j}+s_{j} f\left(x_{j}\right)\right\},
$$

where $0<s_{j}<1$ for $j=1,2, \ldots$, and $\lim _{j \rightarrow \infty} s_{j}=1$. Then the sequence $\left\{x_{j}\right\}$ tends, as $j \rightarrow \infty$, to $\xi$, the Wolff point of $f$. 
Corollary 4.4. Let $D$ be a bounded and strictly convex domain in an arbitrary complex Banach space $(X,\|\cdot\|)$, let $f: D \mapsto D$ be compact, holomorphic $\left(k_{D^{-}}\right.$ nonexpansive) and fixed-point-free, and let $\xi \in \partial D$ be the Wolff point of $f$. For each $z \in D$, let the approximating curve $\{x(s, z): 0<s<1\}$ be defined by

$$
x(s, z)=h_{f}(s, z)=f_{s, z}(x(s, z))=(1-s) z+s f(x(s, z)) .
$$

Then, as $s \rightarrow 1^{-},\{x(s, \cdot)\}$ tends uniformly on $D$ to the constant map taking the value $\xi$.

Corollary 4.5. Let $D$ be a bounded and strictly convex domain in an arbitrary complex Banach space $(X,\|\cdot\|)$, let $f: D \mapsto D$ be compact, holomorphic $\left(k_{D^{-}}\right.$ nonexpansive) and fixed-point-free, and let $\xi \in \partial D$ be the Wolff point of $f$. Let the approximating curve $\{x(s, z): 0<s<1\}$ be defined by

$$
x(s, z)=h_{f}(s, z)=f_{s, z}(x(s, z))=(1-s) z+s f(x(s, z)) .
$$

Then $\lim _{s \rightarrow 1^{-}} \operatorname{diam}_{\|\cdot\|} x(s, D)=0$.

Acknowledgments. The first two authors were partially supported by the Polish MNiSW Grant N N201 393737. The third author was partially supported by the Israel Science Foundation (Grant 389/12), the Fund for the Promotion of Research at the Technion, and by the Technion VPR Research Fund. Part of this research was carried out when the first two authors were visiting the Technion. They are grateful to their colleagues for their kind hospitality.

\section{References}

[1] Abate, M.: Horospheres and iterates of holomorphic maps. - Math. Z. 198, 1988, 225-238.

[2] Aвате, M.: Iteration theory of holomorphic maps on taut manifolds. - Mediterranean Press, 1989.

[3] Budzyńska, M.: A Denjoy-Wolff theorem in $\mathbf{C}^{n}$. - Nonlinear Anal. 75, 2012, 22-29.

[4] Budzyńska, M., T. Kuczumow, and S. Reich: The Denjoy-Wolff theorem for compact holomorphic mappings in reflexive Banach spaces. - J. Math. Anal. Appl. 396, 2012, 504-512.

[5] Budzyńska, M., T. Kuczumow, and S. Reich: Theorems of Denjoy-Wolff type. - Ann. Mat. Pura Appl. (4) (to appear).

[6] CazkA, A.: On conditions under which isometries have bounded orbits. - Colloq. Math. 48, 1984, 219-227.

[7] Chat, S. B.: Holomorphy and calculus in normed spaces. - Marcel Dekker, 1985.

[8] Denjoy, A.: Sur l'itération des fonctions analytiques. - C. R. Acad. Sci. Paris 182, 1926, $255-257$.

[9] Dineen, S.: The Schwarz lemma. - Clarendon Press, 1989.

[10] Dineen, S., and R. M. Timoney: Complex geodesics on convex domains. - In: Progress in functional analysis (Peñíscola, 1990), North-Holland Math. Stud. 170, North-Holland, Amsterdam, 1992, 333-365.

[11] Dineen, S., R. M. Timoney, and J.-P. Vigué: Pseudodistances invariantes sur les domaines d'un espace localement convexe. - Ann. Sc. Norm. Super. Pisa Cl. Sci. (4) 12, 1985, 515-529.

[12] Earle, C. J., and R. S. Hamilton: A fixed point theorem for holomorphic mappings. - Proc. Sympos. Pure Math. 16, Amer. Math. Soc., Providence, RI, 1970, 61-65.

[13] Engelking, R.: Outline of general topology. - Elsevier, 1968. 
[14] Franzoni, T., and E. Vesentini: Holomorphic maps and invariant distances. - NorthHolland, 1980.

[15] Goebel K., and W. A. Kirk: Topics in metric fixed point theory. - Cambridge Univ. Press, 1990.

[16] Goebel, K., and S. Reich: Uniform convexity, hyperbolic geometry, and nonexpansive mappings. - Marcel Dekker, 1984.

[17] Hille, E., and R. S. Philips: Functional analysis and semigroups. - Amer. Math. Soc., Providence, R.I., 1957.

[18] Harris, L. A.: Schwarz-Pick systems of pseudometrics for domains in normed linear spaces. - In: Advances in Holomorphy, North Holland, 1979, 345-406.

[19] JARnicki, M., and P. Pflug: Invariant distances and metrics in complex analysis. - Walter de Gruyter, 1993.

[20] Kapeluszny, J., T. Kuczumow, and S. Reich: The Denjoy-Wolff theorem in the open unit ball of a strictly convex Banach space. - Adv. Math. 143, 1999, 111-123.

[21] Kapeluszny, J., T. Kuczumow, and S. Reich: The Denjoy-Wolff theorem for condensing holomorphic mappings. - J. Funct. Anal. 167, 1999, 79-93.

[22] Kelley, J. L.: General topology. - Springer, 1975.

[23] Kobayashi, S.: Invariant distances on complex manifolds and holomorphic mappings. - J. Math. Soc. Japan 19, 1967, 460-480.

[24] Kobayashi, S.: Hyperbolic manifolds and holomorphic mappings. - Marcel Dekker, 1970.

[25] Kobayashi, S.: Hyperbolic complex spaces. - Springer, 1998.

[26] Kryczka, A., and T. Kuczumow: The Denjoy-Wolff-type theorem for compact $k_{B_{H^{-}}}$ nonexpansive maps on a Hilbert ball. - Ann. Univ. Mariae Curie-Skłodowska Sect. A 51, 1997, $179-184$.

[27] Kuczumow, T., S. Reich, and D. Shoikhet: Fixed points of holomorphic mappings: a metric approach. - In: Handbook of Metric Fixed Point Theory (W. A. Kirk and B. Sims, Eds.), Kluwer Academic Publishers, 2001, 437-515.

[28] Kuczumow, T., and A. Stachura: Iterates of holomorphic and $k_{D}$-nonexpansive mappings in convex domains in $\mathbf{C}^{n}$. - Adv. Math. 81, 1990, 90-98.

[29] Kuczumow, T., and A. Stachura: The Denjoy-Wolff theorem for $s$-condensing mappings. - Ann. Univ. Mariae Curie Skłodowska Sect. A 53, 1999, 109-115.

[30] Lempert, L.: Holomorphic retracts and intrinsic metrics in convex domains. - Anal. Math. 8, $1982,257-261$.

[31] Reich, S., and D. Shoikhet: Nonlinear semigroups, fixed points and geometry of domains in Banach spaces. - Imperial College Press, London, 2005.

[32] Stachura, A.: Iterates of holomorphic self-maps of the unit ball in Hilbert spaces. - Proc. Amer. Math. Soc. 93, 1985, 88-90.

[33] Wolff, J.: Sur l'itération des fonctions holomorphes dans une région, et dont les valeurs appartiennent à cette région. - C. R. Acad. Sci. Paris 182, 1926, 42-43.

[34] Wolff, J.: Sur l'itération des fonctions bornées. - C. R. Acad. Sci. Paris 182, 1926, 200-201.

[35] Wolff, J.: Sur une généralisation d'un théorème de Schwarz. - C. R. Acad. Sci. Paris 182, 1926, 918-920. 